\title{
Markers of renal tubular dysfunction measured annually do not predict risk of microalbuminuria in the first few years after diagnosis of Type I diabetes
}

\author{
C.J. Schultz ${ }^{1}$, R.N. Dalton ${ }^{2}$, H. A.W. Neil ${ }^{1}$, T.Konopelska-Bahu ${ }^{3}$, D. B. Dunger ${ }^{3}$ on behalf of the Oxford Regional \\ Prospective Study Group ${ }^{3}$ \\ ${ }^{1}$ Division of Public Health and Primary Health Care, University of Oxford, Oxford, UK \\ ${ }^{2}$ Children Nationwide Kidney Research Laboratory, Guy's Hospital, London, UK \\ ${ }^{3}$ University Department of Paediatrics, Addenbrooke's Hospital, Cambridge, UK
}

\section{Abstract}

Aims/hypothesis. Early detection of risk of microalbuminuria could prevent early renal damage. We investigated whether urine retinol binding protein and $\mathrm{N}$-acetyl-glucosaminidase could predict the risk of microalbuminuria in a large cohort of children followed from diagnosis of Type I (insulin-dependent) diabetes mellitus.

Methods. Subjects under 16 years of age within a georaphically defined region were recruited at diagnosis of Type I (insulin-dependent) diabetes mellitus. Annually, albumin-, retinol binding protein- and $N$-acetyl-glucosaminidase- to creatinine ratios were each measured in 3 urine samples.

Results. A total of 511 subjects were followed for a median of 6 years (range: 1-14). Microalbuminuria (males: $\geq 3.5 \mathrm{mg} / \mathrm{mmol}$; females: $\geq 4.0 \mathrm{mg} / \mathrm{mmol}$, in 2 out of 3 urines) developed in 78 subjects (36 male). The cumulative probability of microalbuminuria was $40 \%$ after 12 years duration of diabetes. Retinolbinding-proteinuria (men: $\geq 21 \mu \mathrm{g} / \mathrm{mmol}$; women $\geq 33 \mu \mathrm{g} / \mathrm{mmol}$ ) developed in 217 subjects (152 men). The cumulative probability of retinol-binding-proteinuria was $67 \%$ after 12 years duration of diabetes.
The cumulative probability of retinol-binding-proteinuria was $40 \%$ before the onset of microalbuminuria and $59 \%$ in subjects who did not subsequently develop microalbuminuria. Retinol-binding-proteinuria developed at a higher rate with increasing $\mathrm{HbA}_{1 \mathrm{c}}$ than microalbuminuria. $N$-acetyl-glucosaminidase-uria (males: $\geq 56 \mu \mathrm{mol}-\mathrm{pnp} \cdot \mathrm{h}^{-1} \cdot \mathrm{mmol}^{-1}$; females: $\geq 46$ umol-pnp $\cdot \mathrm{h}^{-1} \cdot \mathrm{mmol}^{-1}$ ) developed in 477 subjects. The cumulative probability of $N$-acetylglucosaminidase-uria was $98 \%$ after 10 years of diabetes duration. The cumulative probability of $\mathrm{N}$-acetyl-glucosaminidase-uria was $73 \%$ in the years before the onset of microalbuminuria and $97 \%$ in subjects without microalbuminuria. The probability of $\mathrm{N}$ acetyl-glucosaminidase-uria was $99 \%$ with an $\mathrm{HbA}_{1 \mathrm{c}}$ greater than or equal to $14.5 \%$.

Conclusions/interpretation. Raised amounts of urine retinol binding protein and $N$-acetyl-glycosaminidase are related to $\mathrm{HbA}_{1 \mathrm{c}}$ and the duration of diabetes. They occur in the majority of subjects and are not early markers for the risk of microalbuminuria. [Diabetologia (2001) 44: 224-229]

Keywords Microangiopathy; proximal renal tubular dysfunction, nephropathy, glycated haemoglobin.
Received: 25 July 2000 and in revised form: 29 September 2000

Corresponding author: Prof. D. B. Dunger, Department of Paediatrics, University of Cambridge, Addenbrooks Hospital, Cambridge CB2 2QQ, United Kindom

Abbreviations: ACR, Albumin to creatinine ratio; RBP, retinol binding protein; NAG, $N$-Acetyl-glucosaminidase; RBPCR, retinol binding protein to creatinine ratio; NAGCR, $N$-acetylglucosaminidase to creatinine ratio; ORPS, Oxford Regional Prospective Study.
Macroalbuminuria represents an advanced stage of diabetic renal injury [1]. Microalbuminuria, defined as an albumin excretion rate between $20-200 \mu \mathrm{g} /$ min could predict the development of overt nephropathy in adults with Type I (insulin-dependent) diabetes mellitus [2]. Substantial renal injury, as indicated by changes in renal morphology [3] and incremental rises in blood pressure [4] could, however, already be detectable in subjects with microalbuminuria. 
Progressive glomerular dysfunction is thought to be the primary mechanism for increases in urine protein excretion [5]. However, renal proximal tubule dysfunction could be important in early increases in urine albumin excretion below the threshold of microalbuminuria [5]. Retinol binding protein (RBP), a low molecular weight protein that is freely filtered through the glomerular barrier, is almost completely reabsorbed and catabolised by the renal proximal tubules in healthy subjects [6]. Raised urine RBP is a marker of proximal tubular dysfunction [6]. In children with Type I diabetes urine RBP is positively associated with urine albumin excretion [7], and it has been suggested that raised urine RBP could be an early marker for the risk of incipient nephropathy, although there are few prospective data to confirm this hypothesis.

$\mathrm{N}$-acetyl-glucosaminidase (NAG) occurs in much higher concentrations in the proximal tubules than in other parts of the nephron [8]. High urine levels of this enzyme are considered to be a marker of proximal tubular injury [9]. Raised amounts of NAG in urine could predict the development of microalbuminuria in subjects with Type I diabetes [10] but this observation is controversial $[11,12]$.

We investigated whether raised amounts of urine RBP and NAG are early markers of risk of microalbuminuria in a large cohort of children followed from diagnosis of Type I diabetes in the Oxford Regional Prospective Study.

\section{Subjects and methods}

The study design and methods employed in the Oxford Regional Prospective Study (ORPS) have been reported previously [13]. Briefly, eligible subjects, who were aged under 16 years at the time of diagnosis of Type I diabetes within a geographically defined region, were recruited from the BartsOxford (BOX) diabetes register. Recruitment continued between the years 1986 and 1995. Case-ascertainment was more than $95 \%$ [14] and $91 \%$ of eligible subjects were recruited, with drop-out rates of less than $1 \%$ for each year of study [13]. Subjects diagnosed younger than five years of age were enrolled into the study after a 5 year duration of diabetes, and were assessed annually thereafter. Ethical approval was obtained from district ethics committees. Written informed consent was obtained from parents and children were asked to give assent before the study. The characteristics of 209 children without diabetes (113 female) from whom the normal ranges of urine RBP and NAG were derived have been described previously [15].

At intervals of a year subjects with Type I diabetes were asked to provide three consecutive first void early morning urine specimens to measure the albumin-to-creatinine ratio (ACR), the retinol binding protein-to-creatinine ratio (RBPCR) and the $N$-acetyl- $\beta$-D-glucosaminidase-to-creatinine ratio (NAGCR). Five and ten years after diagnosis three timed, overnight urine samples were collected to estimate the albumin excretion rate (AER). Subjects were followed until they developed overt proteinuria or hypertension except in cases where subjects died or withdrew their co-operation. Urine specimens collected within the first 12 months after diagnosis were excluded from the analysis.

Urine albumin, RBP, NAG and creatinine were measured in a central laboratory. Albumin was measured using a double antibody Enzyme linked Immunosorbent Assay (ELISA) method described previously [13]. The inter-assay coefficient of variation was $12 \%$ at $1.5 \mathrm{mg} / \mathrm{l}$ and $10 \%$ at $16 \mathrm{mg} / \mathrm{l}$. We measured RBP using an ELISA method described previously [16]. The inter-assay coefficient of variation was $3.6 \%$ at $60 \mathrm{mg} / \mathrm{l}$ and $5.7 \%$ at $371.5 \mathrm{mg} / \mathrm{l}$. We measured NAG using an automated colourimetric method, which measures para-nitro-phenol release using the Cobas MIRA spectrophotometer (Roche Diagnostic Systems, Basel, Switzerland) [17]. The inter-assay coefficient of variation was $6.3 \%$ at $2525.3 \mu \mathrm{mol}-\mathrm{pnp} / \mathrm{h}$. Creatinine was measured using the modified Jaffe method (Unimate 7, Roche Diagnostic Systems, Basel, Switzerland) on a Cobas Mira (Roche Diagnostic Systems, Basel, Switzerland) automated spectrophotometer. The coefficient of variation was $2 \%$ at $2.2 \mathrm{mmol} / \mathrm{l}$. Improvements in methodology introduced during the course of the study, such as a change in the storage of urine specimens from $-20^{\circ} \mathrm{C}$ to $-70^{\circ} \mathrm{C}$, and longitudinal quality control have been described previously [13, 15].

$\mathrm{HbA}_{1 \mathrm{c}}$ was also measured centrally. A High Performance Liquid Chromatography (HPLC) method was used from 1992 onward. Details of the methods, methodology changes and quality control have been reported previously [13].

The relation between ACR and AER was determined in 304 timed, overnight urine specimens [13]. Microalbuminuria was defined as the ACR equivalent to an AER of $20 \mu \mathrm{g} / \mathrm{min}$ or more (males: ACR $\geq 3.5 \mathrm{mg} / \mathrm{mmol}$; females: ACR $\geq$ $4.0 \mathrm{mg} / \mathrm{mmol}$ ) but less than $200 \mu \mathrm{g} / \mathrm{min}$ in the median of three consecutive specimens [13]. We definied RBP-uria as RBP/ creatinine ratio (RBPCR) equal to or more than the $97.5^{\text {th }}$ centile in non-diabetic men and women $(21 \mu \mathrm{g} / \mathrm{mmol}$ and $33 \mu \mathrm{g} /$ mmol respectively) in the median of three urine specimens. We definied NAG-uria as a NAG/creatinine ratio (NAGCR) equal to or more than the $97.5^{\text {th }}$ centile in non-diabetic men and women $\left(56 \mu \mathrm{mol}-\mathrm{pnp} \cdot \mathrm{h}^{-1} \cdot \mathrm{mmol}^{-1}\right.$ and $46 \mu \mathrm{mol}-\mathrm{pnp} \cdot \mathrm{h}^{-1}$ - $\mathrm{mmol}^{-1}$ respectively) in the median of three urine specimens.

Statistical methods. The lifetable method was used to calculate the cumulative probability of raised urine proteins over time years of diabetes duration: The logrank test was used to compare cumulative probability distributions. The cumulative probability of raised tubular proteins before the onset of microalbuminuria was calculated by subtracting the diabetes duration at the first appearance of raised tubular proteins from the duration at the first appearance of microalbuminuria. An arbitrary number of 100 was added to the answer to calculate the cumulative distribution curve because the SPSS software programme does not calculate survival probabilities for negative years. Subsequently the arbitrary number was subtracted so that the years before and after the onset of microalbumiuria would be negative and positive respectively. Other statistical methods used were the Chi-squared-test and Spearman's correlation coefficient. Statistical significance was defined as $p$ value of less than 0.05 . The software suite SPSS 7.5 for Windows was used for all analyses.

\section{Results}

Microalbuminuria. A total of 511 subjects (246 male) were examined for a median (range) of 6 years (1 to 14). Microalbuminuria developed in 78 subjects 


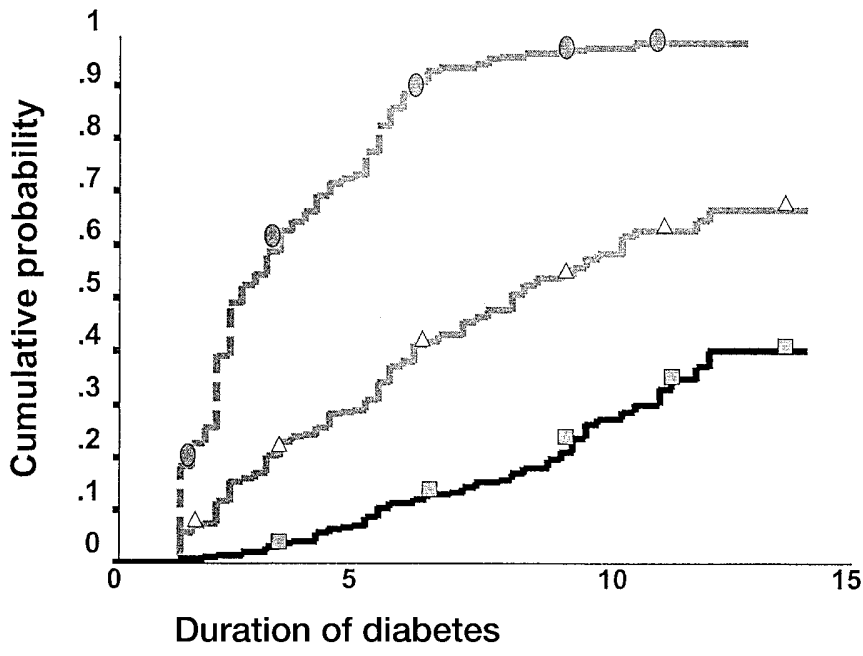

Fig. 1. The cumulative probability (lifetable method) of microalbuminuria $(\square)$, RBP-uria $(\triangle)$ and NAG-uria $(\bigcirc)$ for years from diagnosis of Type I diabetes

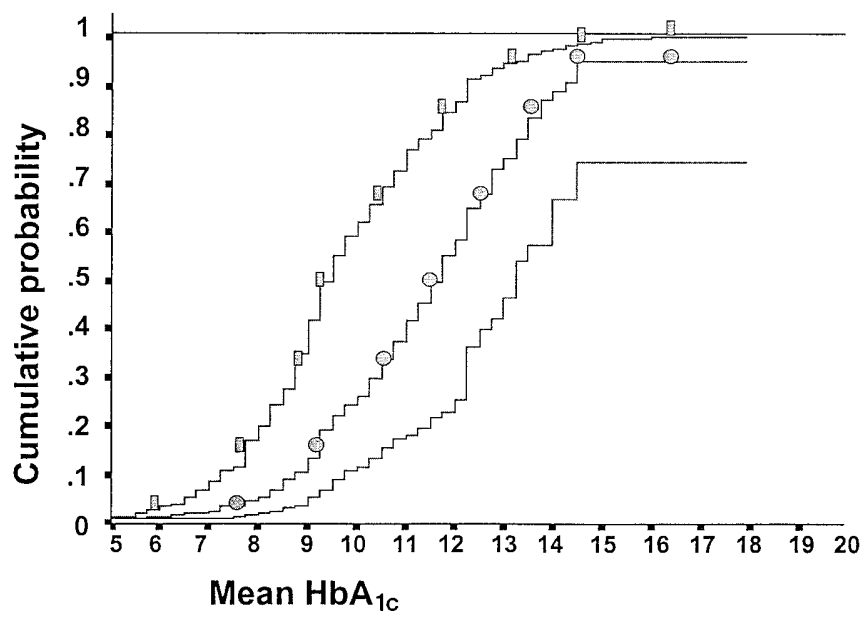

Fig. 2. Cumulative probability (lifetable method) of NAG-uria ( for mean $\mathrm{HbA}_{1 \mathrm{c}}$, as calculated separately from all annual measurements of $\mathrm{HbA}_{1 \mathrm{c}}$ from diagnosis until the development of raised urine levels for each protein

(36 male). The cumulative probability of microalbuminuria, as estimated by the lifetable method, was $40 \%$ after 12 years duration of diabetes (Fig. 1).

The mean of all measurements of $\mathrm{HbA}_{1 \mathrm{c}}$ until onset of microalbuminuria, or the end of follow up, was calculated for each subject. Mean $\mathrm{HbA}_{1 \mathrm{c}}$ was higher in subjects with microalbuminuria compared with those without (mean $\mathrm{HbA}_{1 \mathrm{c}}: 10.5 \%, n=74$ vs $9.7 \%$, $n=415$; mean difference: $-0.8 \% ; 95 \%$ CI of the difference: $-1.3 \%$ to $-0.3 \%$ ). One subject with a mean $\mathrm{HbA}_{1 \mathrm{c}}$ of $5.7 \%$ developed microalbuminuria after 3 years of diabetes. In all other subjects with microalbuminuria the mean $\mathrm{HbA}_{1 \mathrm{c}}$ was $7.3 \%$ or more. The cumulative probability of microalbuminuria increased exponentially with increasing $\mathrm{HbA}_{1 \mathrm{c}}$ (Fig. 2).
A higher proportion of females developed microalbuminuria compared with males although this was not statistically significant (Pearson $\chi^{2}, p=0.08$ ). In non-microalbuminuric subjects the mean $\mathrm{HbA}_{1 \mathrm{c}}$ was similar in males and females $(9.7 \%$ vs $9.6 \%$, $p=0.4)$. In contrast, the mean $\mathrm{HbA}_{1 \mathrm{c}}$ was higher in female compared with male subjects with microalbuminuria, although this was of marginal statistical significance $(10.9 \%, n=40$ vs $10.0 \%, n=34$; mean difference: $0.9 \% ; 95 \% \mathrm{CI}$ of the difference: $1.8 \%$ to $0.0 \%, p=0.06)$.

Raised urine retinol binding protein ( $R B P$-uria). A total of 217 subjects developed RBP-uria (152 males). The cumulative probability of RBP-uria was $67 \%$ after a 12 year duration of diabetes (Fig. 1). The probability of RBP-uria was higher in subjects with microalbuminuria than in those without it $(81 \%$ vs $59 \%$, logrank test $p=0.014$ ). Only $40 \%$ of subjects with microalbuminuria developed RBP-uria in the years before the onset of microalbuminuria. This is in contrast to $59 \%$ of the subjects who developed RBPuria and who did not develop microalbuminuria.

The effect of $\mathrm{HbA}_{1 \mathrm{c}}$ on the development of RBPuria was examined by calculating the mean of all measurements of $\mathrm{HbA}_{1 \mathrm{c}}$ up to the onset of RBP-uria or to the end of follow up, for each individual. The probability of RBP-uria was higher with increasing $\mathrm{HbA}_{1 \mathrm{c}}$ than the probability of microalbuminuria (Fig. 2).

A higher proportion of males compared to females developed RBP-uria in the cohort (54\% vs $18 \%$, Pearson $\left.\chi^{2}: p<0.001\right)$. The probability of RBP-uria was no different in males with microalbuminuria than in those without ( $83 \%$ vs $71 \%$, logrank test: $p=0.09$ ) (Fig. 3). In contrast, the probability of RBP-uria was significantly higher in females with microalbuminuria when compared with those without it (74\% vs $37 \%$, logrank test: $p=0.002)$ (Fig. 3). With further examinations, however, at 10 year duration of diabetes, the cumulative probability of RBP-uria was similar in females with microalbuminuria (74\%) compared with males (Fig.3). The cumulative probabilities (SE) of RBP-uria preceding microalbuminuria in females and males were $29 \%$ (7.0) and $50 \%$ (8.3) respectively.

The effect of puberty on the development of RBPuria was examined by dividing the cohort into three groups by age at diagnosis: (1) 5 years or younger, (2) 5 to 11 years, (3) 11 years or more. Taking into account that in the group younger than 5 years of age urine proteins were not measured until a 5 year duration of diabetes, the incidence distribution of RBPuria was similar in the under 5 years age group, 5 to 11 year age group and 11 year or older groups (Fig.4). A similar proportion of subjects developed RBP-uria in the years before the onset of microalbuminuria in the under 5 years age group, 5 to 


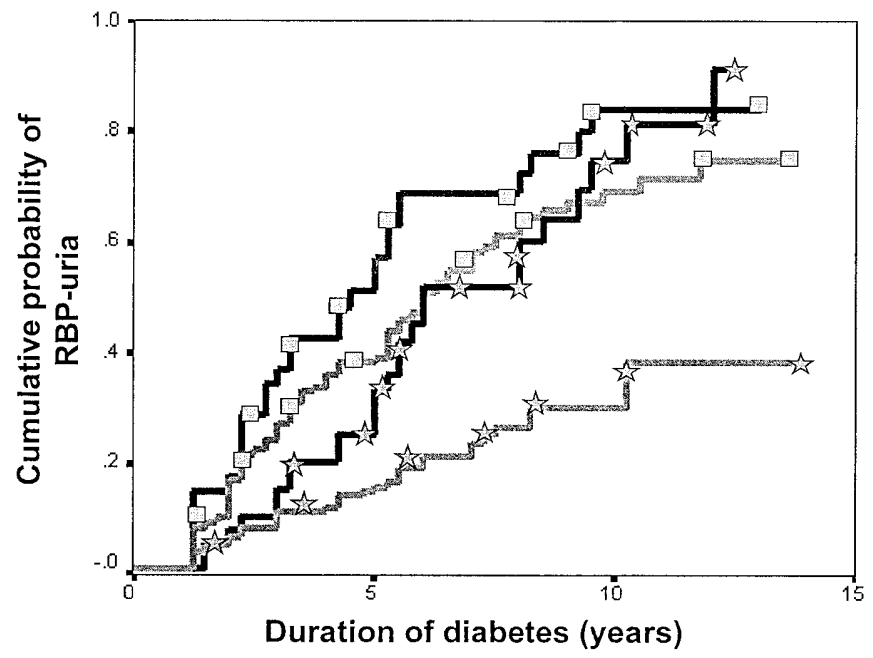

Fig.3. Cumulative probability (lifetable method) of RBP-uria in males $(\square)$ with and without microalbuminuria (black and grey lines respectively), and females ( $\vec{\jmath}$ ) with and without microalbuminuria (black and grey lines respectively), for years from diagnosis of Type I diabetes

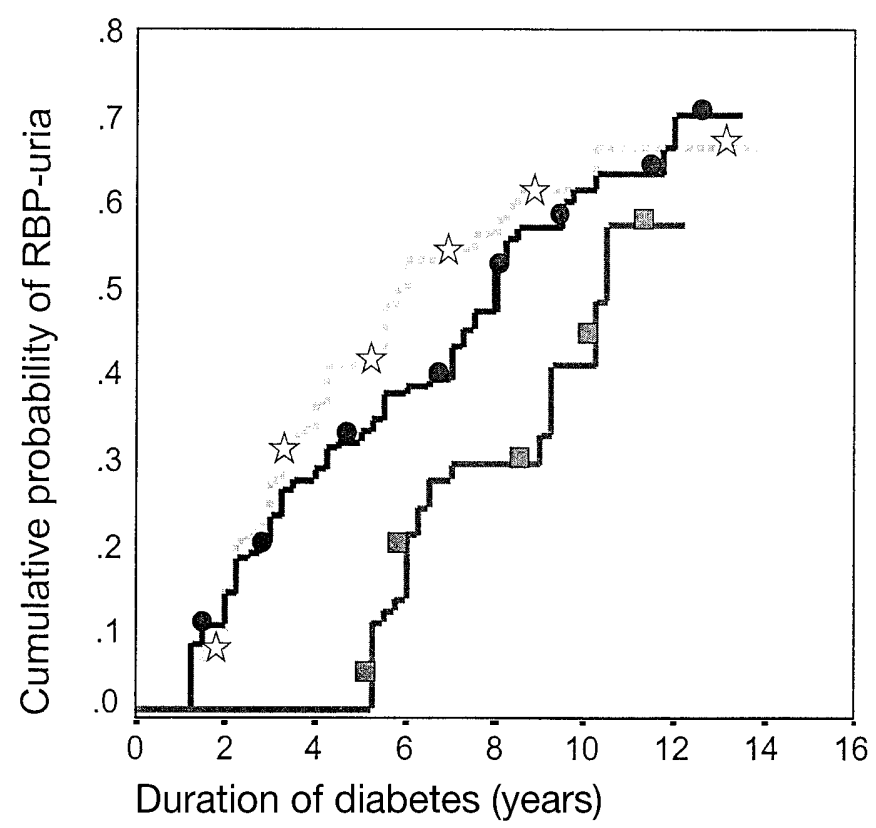

Fig.4. Cumulative probability of RBP-uria across years since diagnosis of Type I diabetes in subjects diagnosed aged under 5 years $(\square), 5$ years to 11 years $(0)$ and $>11$ years $(\underset{\zeta}{ })$

11 year age group and 11 years or older group (42\% vs $37 \%$ vs $39 \%$ ).

Raised urine N-Acetyl-glucosaminidase (NAG-uria). A total of 477 subjects developed NAG-uria. The cumulative probability of NAG-uria was $98 \%$ after a 10 year diabetes duration (Fig. 1). The probability of NAG-uria was similar in subjects with microalbuminuria and in those without it $(98 \%$ vs $97 \%$, logrank: $p=0.8$ ). The cumulative probability of NAG-uria in

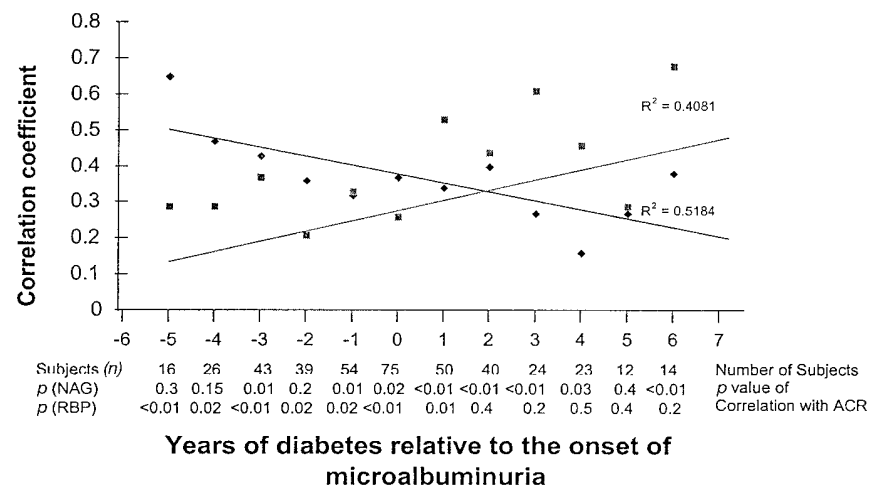

Fig. 5. Correlation coefficients between ACR and NAGCR $(\square)$ and between ACR and RBPCR $(\diamond)$, for each year of diabetes relative to the onset of microalbuminuria (years before and after microalbuminuria are negative and positive respectively), with trendlines (least squares method)

the years before the onset of microalbuminuria was $73 \%$ and this was similar to the probability for those (97\%) who did not develop microalbuminuria.

The effect of $\mathrm{HbA}_{1 \mathrm{c}}$ on the development of NAGuria, increased progressively with higher mean $\mathrm{HbA}_{1 \mathrm{c}}$ (Fig. 2). The probability of NAG-uria was $99 \%$ with an $\mathrm{HbA}_{1 \mathrm{c}}$ of $14.5 \%$ or more (Fig. 2 ).

A slightly higher proportion of females compared with males developed NAG-uria (97\% vs $91 \%$, Pearson $\chi^{2}: p=0.01$ ), but the probability of NAG-uria before the onset of microalbuminuria was similar in males and females (69\% vs $76 \%$, logrank $p=0.9)$. The cumulative probabilities of NAG-uria, in age groups at diagnosis of diabetes of 5 years or younger, 5 to 11 years and 11 years or older were $90 \%, 91 \%$ and $92 \%$, after a 6 year duration of diabetes, respectively.

Increases in urine albumin in parallel with $N A G$ but not $R B P$. We examined the effect of increasing duration of diabetes on associations between urine albumin, RBP and NAG in subjects who developed microalbuminuria. The association between NAGCR and ACR, as estimated by a correlation coefficient, increased between the years before the onset of microalbuminuria and the years after the onset of microalbuminuria (Fig.5). In contrast, the correlation between RBPCR and ACR decreased between the years before the onset of microalbuminuria and the years after the onset of microalbuminuria (Fig.5).

\section{Discussion}

The Oxford Regional Prospective Study (ORPS) is the largest study to date to examine the predictive value of markers of renal tubular function for the risk of microalbuminuria, in subjects with Type I diabetes. 
We found raised NAGCR in about $70 \%$ of subjects before the onset of microalbuminuria but we observed no difference between subjects with microalbuminuria and those without it. In a prospective study in adults, urine NAGCR concentrations were similar at baseline in 9 subjects who developed diabetic nephropathy after 5 years compared with 27 who did not [11]. Similar data have been reported in another small study [12]. In contrast, in a study of adolescents with Type I diabetes, who were followed prospectively for 4.5 years, subjects with raised NAG at baseline had a higher risk of microalbuminuria [10]. In that study [10] the duration of diabetes was, however, significantly shorter in subjects without raised urine NAG compared with those with raised amounts. In our study, the risk of raised NAG and of microalbuminuria rose with the duration of diabetes. Therefore, in the other study in adolescence [10] confounding by differences in duration of diabetes could explain the association of raised NAG with microalbuminuria risk [10]. Furthermore, in that study [10] the majority of subjects with raised NAG did not develop microalbuminuria [10]. These findings indicate that raised NAG is not a marker of risk for microalbuminuria.

The probability of NAG-uria approached $100 \%$ in subjects with insufficient insulinisation as indicated by an $\mathrm{HbA}_{1 \mathrm{c}}$ above $13 \%$. Insufficient insulinisation inhibits glucose uptake by cells, which in turn could inhibit the generation of adenosine triphosphate (ATP). In laboratory models a transient depletion of ATP was followed by apoptosis of renal proximal tubular cells $[18,19]$, which would result in the release of acid hydrolases into urine [8]. These data suggest that raised urine NAG early in the course of Type I diabetes could reflect tubular damage. Furthermore, we observed that NAG increased in parallel with ACR in the years following the onset of microalbuminuria. These findings could reflect increased lysosomal turnover during increased endocytosis of albumin [20-22]. These mechanisms could be investigated further by measuring tissue specific NAG-isoenzymes.

We found RBP-uria was a common occurrence reaching a probability of $67 \%$ after a 12 year duration of diabetes. The probability of RBP-uria in the years before microalbuminuria onset could, however, have been lower than our observations suggested in subjects who remained normoalbuminuric. In a smaller prospective study of the predictive value of RBP, no adolescent with RBP above the normal range subsequently developed microalbuminuria, as measured in spot urine specimens which were regularly collected during the 3-year follow-up period [23]. In another study of 83 adults with Type I diabetes of longer duration, no difference was observed in the amount of RBP at baseline in 13 subjects who had microalbuminuria at follow up 9 years later, when compared with those with normoalbuminuria at both time points [12]. These findings indicate that tubular dysfunction does not always precede microalbuminuria and it frequently occurs in subjects who are at a low risk of nephropathy.

In this study the probability of RBP-uria was higher in males than in females. If the degree of inhibition of tubular function is similar in males and females with Type I diabetes, these differences could relate to the recognised gender difference in glomerular filtration rate (higher in males). Differences in $\mathrm{HbA}_{1 \mathrm{c}}$ and hormonal changes in puberty (sex hormones, growth hormone/IGF-1) could, however, also be important $[24,25]$.

The probability of RBP-uria in subjects with poor glycaemic control was just below $100 \%$. Hypo-insulinaemia could limit the supply of intracellular glucose to mitochondria. As a result, ATP generation rates could be insufficient to sustain protein endocytosis by proximal tubules. Disorders that affect mitochondrial ATP generation are characterised by the Fanconi syndrome [26, 27], a defect of proximal tubular function [26]. These data suggest that RBP-uria could be a marker of ATP-depletion in renal proximal tubule cells in subjects with Type I diabetes. Functional reserve or cell regeneration could be sufficient to compensate for some degree of tubular cell apoptosis, because, in our study, the probability of RBP-uria was lower than the probability of NAGuria at all except the highest levels of $\mathrm{HbA}_{1 \mathrm{c}}$.

Urine RBP and albumin excretion were highly correlated in the years before the onset of microalbuminuria but not in the years afterwards. An increase in vascular and glomerular permeability, a principal mechanism causing microalbuminuria [28], might not be affected by short-term differences in blood glucose concentrations, whereas tubular function could be affected [28]. These data suggest that proximal tubular dysfunction could be important in early increases in urine albumin excretion but that the principal mechanism for microalbuminuria is glomerular dysfunction.

In conclusion, raised urine RBP and NAG are related to $\mathrm{HbA}_{1 \mathrm{c}}$ and the duration of diabetes and occur in the majority of subjects with Type I diabetes. They are not early markers for the risk of microalbuminuria.

Acknowledgements. We would like to thank members of the Oxford Regional Prospective Study Steering Committee are Prof. D. B. Dunger, Dr R. N. Dalton, Prof. J. Fuller, Prof. E.A.M. Gale, Prof. H. Keen, Dr M. Murphy, Dr H. A. W. Neil, Dr C.J. Schultz, Dr R.J. Young and T. Konopelska-Bahu. Members of the Oxford Regional Prospective Study group are doctors J.A.Edge, John Radcliffe Hospital, Oxford, R. A.F Bell, A. Taylor, Horton General Hospital, Banbury; A. Mukthar, B. P. O’Malley, B. R. Silk, E. H. Smith, Kettering District Hospital, Kettering; R.D.M. Scott, King Edward VII Hospital, Windsor; F.M. Ackland, C.J. Fox, N.K. Griffin, 
Northampton General Hospital, Northampton; N. Mann, H. Simpson, P. Cove Smith, M. Pollitzer, Royal Berkshire Hospital, Reading; R.S. Brown, A.H. Knight, Stoke Mandeville Hospital, Aylesbury; J. M. Cowen, J. C. Pearce, Wexham Park Hospital, Slough. The Box Study is co-ordinated by P.J. Bingley, Southmeade Hospital, Bristol. We would like to thank S. Standing for $\mathrm{HbA}_{1 \mathrm{c}}$ data conversion. We acknowledge the continuing help and support of the children and the families who participated in this study; the study field workers, V. Carter, E. Moore as well as L. Barnetson, J. Downey, A. Kingsmill Moore, F. Traynor; the laboratory assistance of A. Watts, D. Harris, T. Carroll and C. Turner; the Barts-Oxford Study fieldworkers and of the paediatricians, physicians and diabetes nurse specialists in the Oxford region. The Oxford Regional Prospective Study is funded by the British Diabetic Association.

\section{References}

1. Mogensen CE (1999) Microalbuminuria, blood pressure and diabetic renal disease: origin and development of ideas. Diabetologia 42: 263-285

2. Viberti GC, Hill RD, Jarrett RJ, Argyropoulos A, Mahmud U, Keen H (1982) Microalbuminuria as a predictor of clinical nephropathy in insulin-dependent diabetes mellitus. Lancet i: 1430-1432

3. Bangstad HJ, Osterby R, Dahl JK, Berg KJ, Hartmann A, Hanssen KF (1994) Improvement of blood glucose control in IDDM patients retards the progression of morphological changes in early diabetic nephropathy. Diabetologia 37: 483-490

4. Poulsen PL, Hansen KW, Mogensen CE (1994) Ambulatory blood pressure in the transition from normo- to microalbuminuria. A longitudinal study in IDDM patients. Diabetes 43: $1248-1253$

5. Abrass CK (1984) Diabetic proteinuria. Glomerular or tubular in origin? Am J Nephrol 4: 337-346

6. Rask L, Anundi H, Bohme J et al. (1980) The retinol-binding protein. Scandi J Clin Lab Invest Suppl 154: 45-61

7. Gibb DMI, Tomlinson PA, Dalton NR, Turner C, Shah V, Barratt TM (1989) Renal tubular proteinuria and microalbuminuria in diabetic patients. Arch Dis Child 64: 129-134

8. Le-Hir M, Dubach UC, Schmidt U (1979) Quantitative distribution of lysosomal hydrolases in the rat nephron. Histochemistry 63: 245-251

9. Kunin CM, Chesney RW, Craig WA, England AC, DeAngelis C (1978) Enzymuria as a marker of renal injury and disease: studies of $\mathrm{N}$-acetyl beta-glucosaminidase in the general population and in patients with renal disease. Pediatrics 62: 751-760

10. Kordonouri O, Hartmann R, Muller C, Danne T, Weber B (1998) Predictive value of tubular markers for the development of microalbuminuria in adolescents with diabetes. Horm Res 50 [Suppl 1]: 23-27

11. Agardh CD, Tallroth G, Hultberg B (1987) Urinary Nacetyl-beta-D-glucosaminidase activity does not predict development of diabetic nephropathy. Diabetes Care 10: 604-606

12. O'Brien SF, Powrie JK, Watts GF (1997) Comparison of urinary albumin, retinol-binding protein and $\mathrm{N}$-acetyl beta-glucosaminidase as predictors of progression of low level albuminuria in diabetes. Ann Clin Biochem 34: 202-204
13. Schultz CJ, Konopelska-Bahu T, Dalton RN et al. (1999) Microalbuminuria prevalence varies with age, sex and puberty in children with insulin dependent diabetes followed in a longitudinal study from diagnosis. Diabetes Care 22: 495-502

14. Gardner SG, Binctley PJ, Sawtell PA et al. (1997) Rising incidence of insulin dependent diabetes in children aged under 5 years in the Oxford region: time trend analysis. The Bart's-Oxford Study Group. BMJ 315: 713-717

15. Schultz CJ, Dalton RN, Turner C, Neil HA, Dunger DB (2000) Freezing method affects the concentration and variability of urine proteins and the interpretation of data on microalbuminuria. The Oxford Regional Prospective Study Group. Diabet Med 17: 7-14

16. Tomlinson PA, Dalton RN, Turner C, Chantler C (1990) Measurement of beta 2-microglobulin, retinol-binding protein, alpha 1-microglobulin and urine protein 1 in healthy children using enzyme-linked immunosorbent assay. Clin Chim Acta 192: 99-106

17. Maruhn D (1976) Rapid colorimetric assay of beta-galactosidase and N-acetyl-beta- glucosaminidase in human urine. Clin Chim Acta 73: 453-461

18. Wang Y, Knowlton AA, Christensen TG, Shih T, Borkan SC. (1999) Prior heat stress inhibits apoptosis in adenosine triphosphate-depleted renal tubular cells. Kidney Int 55: 2224-2235

19. Shanley PF, Brezis M, Spokes K, Silva P, Epstein FH, Rosen S (1986) Differential responsiveness of proximal tubule segments to metabolic inhibitors in the isolated perfused rat kidney. Am J Kidney Dis 7: 76-83

20. Lockwood TD, Bosmann HB (1979) The use of urinary Nacetyl-beta-glucosaminidase in human renal toxicology. 1. Partial biochemical characterization and excretion in humans and release from the isolated perfused rat kidney. Toxicol Appl Pharmacol 49: 323-336

21. Mahadevan S, Dillard CJ, Tappel AL (1969) Degradation of polysaccharides, mucopolysaccharides, and glycoproteins by lysosomal glycosidases. Arch Biochem Biophys 129: 525-533

22. Bosomworth MP, Aparicio SR, Hay AW (1999) Urine Nacetyl-beta-D-glucosaminidase - a marker of tubular damage? Nephro DiaI Transplant 14: 620-626

23. Ginevri F, Piccotti E, Alinovi R et al. (1993) Reversible tubular proteinuria precedes microalbuminuria and correlates with the metabolic status in diabetic children. Pediatr Nephrol 7: 23-26

24. Whitman MM, Harnish DC, Soprano KJ, Soprano DR (1990) Retinol-binding protein mRNA is induced by estrogen in the kidney but not in the liver. J Lipid Res 31: 1483-1490

25. Ahluwalia GS, Kaul L, Ahluwalia BS (1980) Evidence of facilitory effect of growth hormone on tissue vitamin A uptake in rats. J Nutr 110: 1185-1193

26. Niaudet P, Rotig A (1996) Renal involvement in mitochondrial cytopathies. Pediatr Nephrol 10: 368-373

27. Di-Cataldo A, Palumbo M, Pittala D et al. (1999) Deletions in the mitochondrial DNA and decrease in the oxidative phosphorylation activity of children with Fanconi syndrome secondary to antiblastic therapy. Am J Kidney Dis 34: $98-106$

28. Feldt Rasmussen B (1986) Increased transcapillary escape rate of albumin in Type I (insulin-dependent) diabetic patients with microalbuminuria. Diabetologia 29: 282-286 